\title{
Strain-dielectric response of dielectrics as foundation for electrostriction stresses
}

\author{
Ho Young Lee \\ Department of Electrical and Computer Engineering, University of Wisconsin, Madison, Wisconsin 53706 \\ Yiyan Peng \\ Department of Mechanical Engineering, University of Wisconsin, Madison, Wisconsin 53706 \\ Yuri M. Shkel ${ }^{\mathrm{a}}$ \\ Department of Mechanical Engineering, Department of Electrical and Computer Engineering, University \\ of Wisconsin, Madison, Wisconsin 53706
}

(Received 29 March 2005; accepted 22 August 2005; published online 11 October 2005)

\begin{abstract}
The apparent deformation due to an electric field does not rigorously describe the electrostriction phenomenon. This is in part due to uncertainties in the mechanical constraints at the specimen boundaries. Such constraints are very critical in thin films. Determining the electric-field-induced stress seems to be a more adequate approach to electrostriction. General thermodynamic considerations identify the electrostriction stress through strain derivatives of the dielectric displacement. Consequently, the derivatives of the dielectric coefficients are termed the electrostriction parameters. The strain-dielectric response of a material, which is called dielectrostriction, provides an avenue to study electrostriction. However, controlling the mechanical boundary conditions of a thin-film specimen can be challenging. This problem can be overcome by using a proposed planar capacitor sensor which does not require any mechanical contact with the specimen. The theoretical background and experimental results for the dielectrostriction study of a uniaxially loaded specimen are presented and discussed. (C) 2005 American Institute of Physics.
\end{abstract} [DOI: $10.1063 / 1.2073977$ ]

\section{INTRODUCTION}

\section{A. Electrostatic stress as manifestation of electrostriction}

Electrostriction is the deformation of a dielectric under forces exerted by an electrostatic field. ${ }^{1}$ It is commonly treated phenomenologically as a fourth-rank tensor of electrostrictive coefficients $\gamma_{i k l m}$, which links the strain tensor $u_{i k}$ $\left[=\left(\partial u_{i} / \partial x_{k}+\partial u_{k} / \partial x_{i}\right) / 2\right.$, where $\mathbf{u}(\mathbf{x})$ is the displacement field $]$ to the electric field $\mathbf{E},{ }^{2} u_{i k}=\gamma_{i k l m} E_{l} E_{m}$. Devonshire ${ }^{3}$ proposed another phenomenological description with polarization $\mathbf{P}$, as the state variable. This leads to an equivalent form $u_{i k}$ $=Q_{i k l m} P_{l} P_{m}$, where $Q_{i k l m}$ is the fourth-rank electrostriction tensor. For fixed boundary conditions this tensor can be expressed and measured through derivative of dielectric stiffness with stresses. ${ }^{4,5}$ However, this derivative depends on the constraints at the specimen boundaries. The electrostriction tensor $\gamma_{i k l m}$ (or $Q_{i k l m}$ ) can therefore vary from one experimental setup to another. This makes a different approach where electrostriction is described through field-induced stresses more suitable. ${ }^{6,7}$

Following Landau and Lifshitz, the total stress in an elastic material can be expressed in terms of the free energy per unit volume, $\widetilde{F}\left(u_{i k}, T, \mathbf{E}\right)$, where that energy is the thermodynamic potential of three thermodynamic parametersthe strains $u_{i k}$, temperature $T$, and the electric field $\mathbf{E}$,

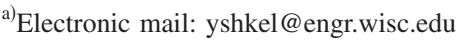

$$
\sigma_{i k}=\tilde{F} \delta_{i k}+\left.\frac{\partial \widetilde{F}}{\partial u_{i k}}\right|_{T, \mathbf{E}}+\frac{E_{i} D_{k}+E_{k} D_{i}}{2} .
$$

The relation $E_{i} D_{k}=E_{k} D_{i}$ simplifies Eq. (1) for isotropic dielectrics. Total free energy is the energy without an electric field, $F_{0} \equiv \widetilde{F}\left(u_{i k}, T, \mathbf{E}=0\right)$, and an additional term due to polarization, $-\int \mathbf{D} \cdot d \mathbf{E}$,

$$
\tilde{F}\left(u_{i k}, T, \mathbf{E}\right)=F_{0}-\int_{0}^{E} \mathbf{D} \cdot d \mathbf{E} .
$$

The dielectric displacement $\mathbf{D}$ is related to $\mathbf{E}$ by the secondorder dielectric tensor $\varepsilon_{i k}$ as $D_{i}=\varepsilon_{0} \varepsilon_{i k} E_{k}$, where $\varepsilon_{0}$ is the permittivity of free space. Even an initially isotropic material becomes anisotropic when deformed. The dielectric tensor $\varepsilon_{i k}$ is a function of the strain $u_{i k}$ and the temperature $T$. In general, the polarizability of a material is also affected by the applied electric field $\mathbf{E}$. Such nonlinear behavior can be observed in some electroactive materials. However, for many dielectrics and electric-field strengths of interest, the dielectric tensor is independent of the electric field (so-called "linear dielectrics"). The integral in Eq. (2) can be solved for a linear dielectric material and substituted into Eq. (1). The total stress $\sigma_{i k}$ consists of the mechanical (e.g., elastic) stress $\sigma_{i k}^{\text {mech }}$ and the electrostatic stress $\sigma_{i k}^{\text {elect }}$. The mechanical stress $\sigma_{i k}^{\text {mech }}$ is the stress in the absence of an electric field and is determined by $F_{0}=\widetilde{F}\left(u_{i k}, T, \mathbf{E}=0\right)$ which is part of the free energy, 
(a)

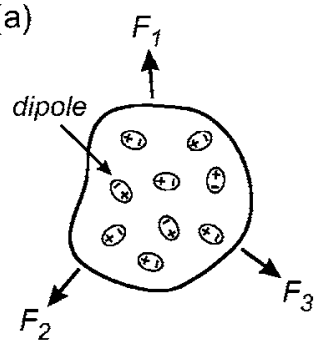

(b)

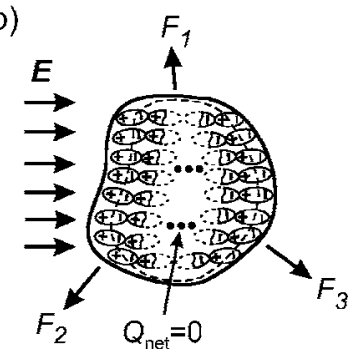

FIG. 1. (a) $E=0$ : electric dipoles are randomly oriented and the material response to load described by its mechanical stress $\sigma_{i k}^{\text {mech }}$; (b) $E \neq 0$ : bound charges appear on the surface of the material, and dipoles are oriented along the field.

$$
\sigma_{i k}^{\text {mech }}=F_{0} \delta_{i k}+\left.\frac{\partial F_{0}}{\partial u_{i k}}\right|_{T} .
$$

Applying an electric field introduces electrostatic stress $\sigma_{i k}^{\text {elect }}$ into the total stress. The following expression for the total stress is valid for both isotropic and anisotropic linear dielectric materials: ${ }^{8}$

$$
\sigma_{i k}=\sigma_{i k}^{\mathrm{mech}}-\frac{\mathbf{E} \cdot \mathbf{D}}{2} \delta_{i k}+\frac{E_{i} D_{k}+E_{k} D_{i}}{2}-\left.\frac{1}{2} \frac{\partial D_{l}}{\partial u_{i k}}\right|_{T, \mathbf{E}} E_{l} .
$$

Three distinctive contributions to the total stress $\sigma_{i k}$ can be identified: (a) mechanical stress $\sigma_{i k}^{\text {mech }}$ which for linear-elastic materials is determined by Hooke's law, (b) the Maxwell stress $\left(-\mathbf{E} \cdot \mathbf{D} \delta_{i k}+E_{i} D_{k}+E_{k} D_{i}\right) / 2$, and (c) electrostriction stress $-\left.\left(\partial D_{l} / \partial u_{i k}\right)\right|_{T, \mathbf{E}} E_{l} / 2$. The response of a material to mechanical loading, with and without the application of an electric field (see Fig. 1), reveals each contribution to the total stress. If $E=0$, the material demonstrates pure elastic behavior, $\sigma_{i k}^{\text {mech }}$, which is described, for example, by Hooke's law. In addition, the material is electrically neutral and all electric dipoles composing the material are randomly oriented. Now, if an electric field is applied, $E \neq 0$, the following major changes in the material's state occur: (1) bound charges appear on its surface and (2) dipoles within its volume are oriented along the field. The Coulomb attraction of the bound charges is described by the Maxwell stress tensor, ${ }^{1,8}$ which is the second term in Eq. (3). Some researchers consider the Maxwell stress more like a mathematical formulation which represents the surface effects of an electric field rather than a real stress existing at a given point of a solid body. ${ }^{9}$ Deformations due to the Coulomb attraction of surface charges are not distinct from those of any external load and according to Stratton ${ }^{1}$ should not be called electrostriction. Another effect, the field-induced orientation of dipoles, affects the material structure and its elastic response. This so-called electrostriction stress manifests itself as an additional field-induced stress and as an alteration of the material's elastic properties. Shkel and Klingenberg ${ }^{10}$ derived the electrostriction stress for uniaxially isotropic dielectrics and identified both contributions - an additional field-induced electrostatic stress and an electrorheological effect when the shear modulus becomes a function of the applied field. According to Eq. (3) the electrostriction stress can be determined from the derivatives of the material's dielectric coefficient with respect to

deformations. The variation of dielectric properties with deformation is the fundamental phenomenon observed in any dielectric material. To avoid confusion with the already wellaccepted term "electrostriction," the strain effect on the dielectric properties of a material will be called dielectrostriction. Dielectrostriction has a physical basis very similar to that of the photoelastic effect. Moreover, photoelasticity can be considered as a manifestation of dielectrostriction in the optical range of electromagnetic spectrum.

\section{B. Dielectrostriction}

Dielectrostriction is defined as the variation of the dielectric properties of a material with deformations. ${ }^{11}$ The relationship between strains and polarization introduces a set of material parameters which determine the field-induced stresses and are called electrostriction parameters. ${ }^{1,8}$ It would be instructive to review the relationship between dielectrostriction and electrostriction for isotropic materials where a single constant $\varepsilon$ describes the dielectric properties. For consistency with future considerations, the dielectric properties of such materials will be represented as a secondrank tensor, $\varepsilon_{i k}^{0}=\varepsilon \delta_{i k}$, where $\delta_{i k}$ is the Kronecker delta. Arbitrary deformation would introduce anisotropy into the structure of the initially isotropic material and its dielectric tensor $\varepsilon_{i k}$ would become 8

$$
\varepsilon_{i k}=\varepsilon \delta_{i k}+\alpha_{1} u_{i k}+\alpha_{2} u_{l l} \delta_{i k}
$$

This is the most general linear relation between deformations and dielectric properties and it introduces two new dielectric parameters $\alpha_{1}$ and $\alpha_{2}$. In this index notation, $u_{l l}\left(\equiv u_{x x}+u_{y y}\right.$ $\left.+u_{z z}\right)$ is the first strain invariant and describes a relative change in the volume $\Delta V / V$. Substitution of Eq. (4) into Eq. (3) yields the well-known expression for the electrostatic stress $\sigma_{i k}^{\text {elect }}$ in isotropic solid dielectrics, ${ }^{8}$

$$
\sigma_{i k}^{\text {elect }}=\frac{1}{2} \varepsilon_{0}\left(2 \varepsilon-\alpha_{1}\right) E_{i} E_{k}-\varepsilon_{0}\left(\varepsilon+\alpha_{2}\right) E^{2} \delta_{i k} .
$$

According to the above definitions, this equation describes the dielectrostriction phenomenon, and the material parameters $\alpha_{1}$ and $\alpha_{2}$ are called the electrostriction parameters because they determine the electrostriction stress in an isotropic material. One can estimate these electrostriction parameters for a suspension of randomly distributed polarizable inclusions. ${ }^{12}$ If the polarization of the matrix is negligible compared with the contribution of inclusions, both parameters $\alpha_{1}$ and $\alpha_{2}$ can be expressed through the composite's dielectric constant $\varepsilon$ as

$\alpha_{1}=-\frac{2}{5}(\varepsilon-1)^{2}$ and $\alpha_{2}=-\frac{1}{3}(\varepsilon-1)(\varepsilon+2)+\frac{2}{15}(\varepsilon-1)^{2}$.

The contribution of electrostriction to the total electrostatic stress exceeds the Maxwell stress in materials having dielectric constants $\varepsilon \geqslant 4$. Therefore the electrostriction parameters are important and should be measured for any candidate electroactive material. 
(a)

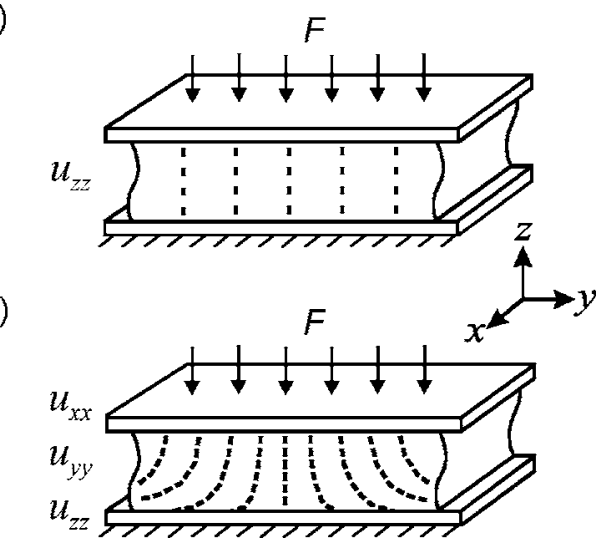

FIG. 2. A thin film is placed between two rigid electrodes. The dashed lines represent the displacement field due to applied load. (a) The film attached to the electrodes is compressed only in the $z$ direction; (b) the film, which can slip between the electrodes, is compressed in the $z$ direction and is able to expand in the $x y$-plane directions.

\section{DIELECTROSTRICTION STUDY}

\section{A. Synergy of dielectricity and elasticity}

A traditional specimen for dielectric study represents a thin film of thickness $h$ having deposited or attached electrodes of area $A$. A capacitance, $C=\varepsilon_{0} \varepsilon A / h$, of this parallelplate configuration can be measured by a variety of methods. ${ }^{13}$ However, elastic measurements are difficult for such a thin-film configuration because of uncertainties about the mechanical conditions at its boundaries. An applied load would deform the material, affect its dielectric properties, and possibly change the specimen area. Moreover, different constraints on the specimen's boundaries would result in different strains under the same load. Therefore, the apparent dielectrostriction response would also vary with the boundary constraints. For example, a variation of the film dielectric properties, $\Delta \varepsilon=\alpha_{1} \Delta h / h+\alpha_{2} \Delta V / V$, occurs due to the variation in film thickness $\Delta h$ and its volume $\Delta V$. Parameter $\alpha_{1}$ is associated with the change in dielectric properties of the material due to a linear deformation, while $\alpha_{2}$ accounts for any volume change. Both material parameters $\alpha_{1}$ and $\alpha_{2}$ can be obtained with two measurements having different contributions of $\Delta h / h$ and $\Delta V / V$. This goal could be achieved with the two types of boundary conditions shown in Fig. 2. A load $F$ compresses the film between two rigid electrodes. Figure 2(a) shows the film attached to the electrodes such that it can be compressed only in $z$ direction $u_{z z}$. The film in Fig. 2(b) can slip between electrodes-it is compressed in $z$ direction $u_{z z}$ and expands in the plane directions, $u_{x x}=u_{y y}=-\nu u_{z z}$, where $\nu$ is Poisson's ratio. For the boundary conditions in Fig. 2(a) the linear and the volume deformations are equal to each other, $\Delta h / h=\Delta V / V=u_{z z}$, and so the dielectrostriction response for small deformations can be represented as

$$
\alpha_{1}+\alpha_{2}=\frac{d \varepsilon_{z z}}{d u_{z z}} .
$$

However, for the boundary conditions in Fig. 2(b), the film inclines preserve its volume and $\Delta V / V=u_{x x}+u_{y y}+u_{z z}$ $=(1-2 \nu) u_{z z}$. Recognizing that materials such as polymers might have low compressibility (Poisson's ratio $\nu \approx 0.5$ ), the volume effect can be very small. The dielectrostriction response of an unconstrained film can be represented as

$$
\alpha_{1}+\alpha_{2}(1-2 \nu)=\frac{d \varepsilon_{z z}}{d u_{z z}} .
$$

Thus, if $d \varepsilon_{z z} / d u_{z z}$ were measured for both of the above configurations, $\alpha_{1}$ and $\alpha_{2}$ could be determined by solving Eqs. (6) and (7) simultaneously. However, in real life, it would be very difficult to control constraints at the surfaces of a thin film. In other words, a thin-film polymeric sample exhibits a dielectrostriction response somewhere between those predicted by expressions (6) and (7). Shkel and Klingerberg ${ }^{6}$ obtained the electrostriction parameters $\alpha_{1}$ and $\alpha_{2}$ by measuring the strain derivative $d \varepsilon_{z z} / u_{z z}$ for different loads on the top plate. It was assumed that friction between the electrodes and the film increases with the load. Conditions corresponding to expressions (6) and (7) are identified as two limiting values of $d \varepsilon_{z z} / d u_{z z}$. Equation (7) was applied for a nearly zero load and Eq. (6) was used for the load approaching infinity.

Shear deformation of a film between two parallel electrodes is another desirable configuration having well-defined boundary conditions. Kim and Shkel ${ }^{14}$ used a rheometer to study shear dielectrostriction of a silicone resin-based uniaxially isotropic composite. The specimen geometry was unaffected by rotation of the rheometer plate and the output signal was directly proportional to the dielectrostriction response. This experimental setup provides only one combination of the electrostriction parameters but this is sufficient to describe the electrorheological effect.

\section{B. Planar capacitor sensor}

The dielectric tensor $\boldsymbol{\varepsilon}$ of an anisotropic dielectric material has three principal dielectric constants $\varepsilon_{1}, \varepsilon_{2}$, and $\varepsilon_{3}$ in the principal coordinate system $x_{1} x_{2} x_{3}$ and can be represented as

$$
\boldsymbol{\varepsilon}=\left[\begin{array}{ccc}
\varepsilon_{1} & 0 & 0 \\
0 & \varepsilon_{2} & 0 \\
0 & 0 & \varepsilon_{3}
\end{array}\right] .
$$

A planar capacitor sensor consists of a system of interdigitated electrodes deposited on a rigid substrate (see Fig. 3). Electrodes are in close proximity but not attached to the dielectric material and therefore do not mechanically constrain the dielectric. All electrodes of the planar capacitor have width $2 a$ and are equally separated by $2 a$. Both the thickness of the dielectric layer, $h$, and the length of the electrodes, $l$, are much larger than the distance between the electrodes $(h / 2 a, l / 2 a \gg 1)$. The electrodes are located in the $x_{1} x_{2}$ plane and form an arbitrary angle $\theta$ with the principal axis $x_{2}$ of the dielectric material. The capacitance of the interdigitated electrodes with attached anisotropic material will be calculated after a sequence of simplified assumptions.

\section{Capacitance of interdigitated electrodes}

It is instructive to first consider the electrode configuration in Fig. 4 which allows for an exact analytical solution. 
(a)

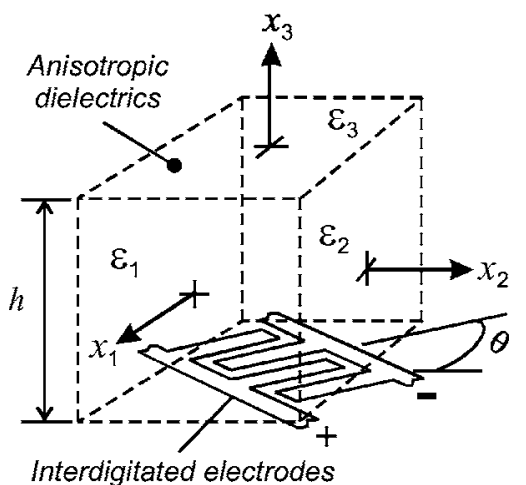

(b)

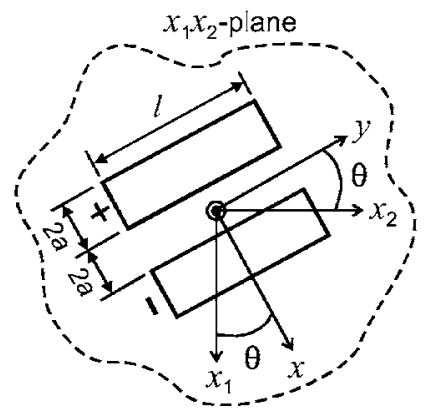

FIG. 3. (a) An anisotropic solid dielectric has three principal dielectric constants, $\varepsilon_{1}, \varepsilon_{2}$, and $\varepsilon_{3}$, which are defined in the principal coordinate system $x_{1} x_{2} x_{3}$. Interdigitated electrodes are located in the $x_{1} x_{2}$ plane and form an angle $\theta$, with the principal axis $x_{2}$. (b) Each electrode strip has width $2 a$, length $l$, and is separated from the other electrodes by $2 a$.

Figure 4 shows two electrodes with each covering half of the plane and almost touching the other. One electrode has the electrical potential $\phi_{1}$ and the other has the potential $\phi_{2}$. The overall electrode length $L$ equals the total length of all the individual strips in Fig. 3. An anisotropic material is located on top of the electrodes of Fig. 4. The capacitance $C$ of any system of electrodes is defined as the ratio $C=Q / V$, where $Q$ is the charge needed to produce the potential difference $V$ $=\phi_{1}-\phi_{2}$. The surface density of the charge, $\rho_{s}$, can be expressed through the normal component of the dielectric displacement, $D_{n}$, on the electrode surface, as $\rho_{s}=D_{n 1}+D_{n 2}$. The dielectric displacements $D_{n 1}$ and $D_{n 2}$ are taken at the same locations but on opposite sides of the electrode surfaces. The electrode strip having length $L$ and width $d r$ car-

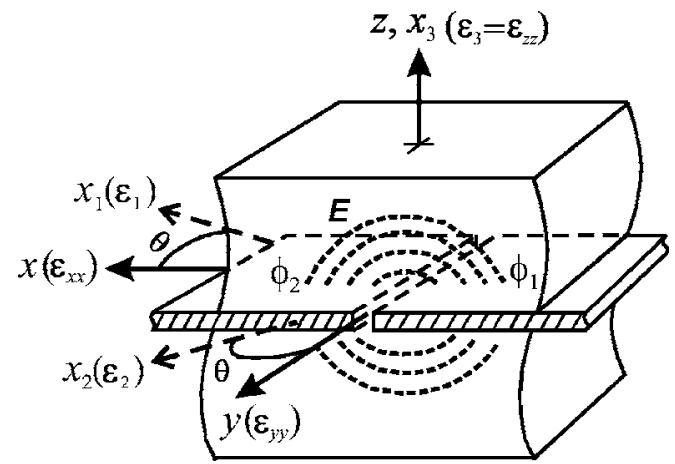

FIG. 4. Each electrode covers half of the plane and almost touches the other electrode along the $y$ axis. One electrode has the electrical potential $\phi_{1}$ and the other has the potential $\phi_{2}$. The overall electrode length is $L$. An anisotropic material is located on both sides of the electrodes. ries the charge $d Q\left(=\rho_{s} L d r\right)$. The electric-field potential $\phi$ $(\mathbf{E}=-\nabla \phi)$ is described by Maxwell's equations with the dielectric tensor $\boldsymbol{\varepsilon}$ defined by Eq. (8), $\nabla \cdot \boldsymbol{\varepsilon} \cdot \nabla \phi=0$. In the principal coordinate system $x_{1} x_{2} x_{3}$, this equation has the form

$$
\varepsilon_{1} \frac{\partial^{2} \phi}{\partial x_{1}^{2}}+\varepsilon_{2} \frac{\partial^{2} \phi}{\partial x_{2}^{2}}+\varepsilon_{3} \frac{\partial^{2} \phi}{\partial x_{3}^{2}}=0 .
$$

This equation can be converted into the well-known Laplace equation by scaling the coordinate axes $X_{1}=x_{1} / \sqrt{ } \varepsilon_{1}, X_{2}$ $=x_{2} / \sqrt{ } \varepsilon_{2}$, and $X_{3}=x_{3} / \sqrt{ } \varepsilon_{3}$ as $^{2,8}$

$$
\frac{\partial^{2} \phi}{\partial X_{1}^{2}}+\frac{\partial^{2} \phi}{\partial X_{2}^{2}}+\frac{\partial^{2} \phi}{\partial X_{3}^{2}}=0 \text {. }
$$

Equation (9) can be solved in the $X_{1} X_{2} X_{3}$-coordinate system and the obtained solution is then converted back to the principal coordinate system. As an intermediate step the coordinate system $X_{1} X_{2} X_{3}$ can be rotated around the $X_{3}$ axis through the angle $\theta$. If the electrodes are parallel to the $y$ axis of the $x y z$-coordinate system then the electric-field potential is expressed as

$$
\phi=\phi_{1}+\frac{\phi_{2}-\phi_{1}}{\pi} \tan ^{-1}\left(\sqrt{\frac{\varepsilon_{x x}}{\varepsilon_{z z}}} \frac{z}{x}\right) .
$$

Consequently, the normal component of the dielectric displacement at the electrode surface, $D_{n}$, is obtained from the relation $\mathbf{D}=-\varepsilon_{0} \boldsymbol{\varepsilon} \cdot \nabla \phi$ using the above $\phi$,

$$
D_{n}=\frac{\varepsilon_{0} \sqrt{\varepsilon_{x x} \varepsilon_{z z}}}{\pi r}\left(\phi_{1}-\phi_{2}\right) \text {. }
$$

Here $r$ is the distance from the midpoint between the electrodes to a point on the electrode surface, while $\varepsilon_{x x}$ and $\varepsilon_{z z}$ are the components of the dielectric tensor in the $x y z$-coordinate system. Finally, the potential and the component of dielectric displacement can be expressed in the principal coordinate system $x_{1} x_{2} x_{3}$ as

$$
\begin{aligned}
\phi= & \phi_{1}+\frac{\phi_{2}-\phi_{1}}{\pi} \\
& \times \tan ^{-1}\left\{\sqrt{\frac{\left(\varepsilon_{1}+\varepsilon_{2}\right) / 2+\left(\varepsilon_{1}-\varepsilon_{2}\right) \cos 2 \theta}{2 \varepsilon_{3}}}\right. \\
& \left.\times \frac{x_{3}}{x_{1} \cos \theta+x_{2} \sin \theta}\right\}, \\
D_{n}= & \frac{\varepsilon_{0}}{\pi r} \sqrt{\varepsilon_{3}\left[\left(\frac{\varepsilon_{1}+\varepsilon_{2}}{2}\right)+\left(\frac{\varepsilon_{1}-\varepsilon_{2}}{2}\right) \cos 2 \theta\right]\left(\phi_{1}-\phi_{2}\right),}
\end{aligned}
$$

where $\varepsilon_{1}, \varepsilon_{2}$, and $\varepsilon_{3}$ are the principal values of the dielectric tensor. Once $D_{n}$ is known, the charge density at the electrode surface, $\rho_{s}$, can be determined and the total charge $Q$ over the entire electrode surface can be calculated. We will assume that the solution given by Eq. (12) is also valid for the planar capacitor sensor in Fig. 3. Due to the symmetry of the field distribution near the electrodes, the whole system can be modeled as just two electrodes, each of width $a$ and total length $L$. Finally, the capacitance $C_{\theta}$ of a planar capacitor 


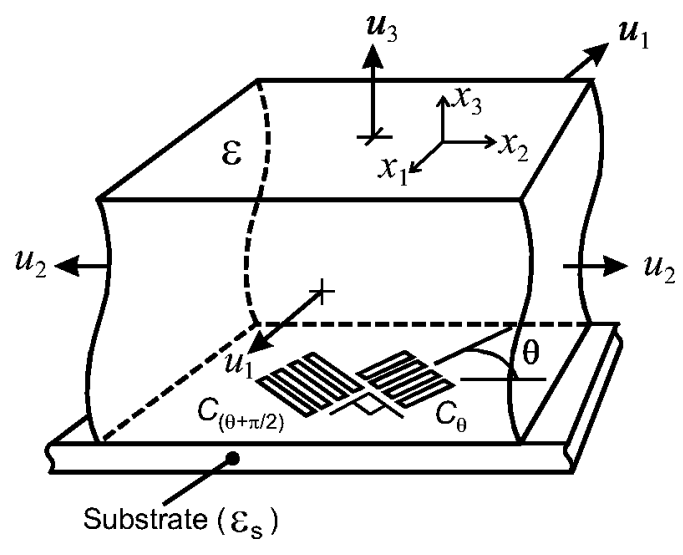

FIG. 5. An elastic dielectric is subjected to three axial strains, $u_{1}, u_{2}$, and $u_{3}$, which are the principal strains in the principal coordinate system $x_{1} x_{2} x_{3}$. Two planar capacitor sensors form angles $\theta$ and $(\theta+\pi / 2)$ with the $x_{2}$ axis, respectively.

sensor in the $x_{1} x_{2}$ plane at an arbitrary angle $\theta$ with the principal axis $x_{2}$ is determined as the ratio between the total charge of the electrode surface and the potential difference, $V=\phi_{1}-\phi_{2}$,

$$
\begin{aligned}
& C_{\theta}=\frac{Q}{V}=C_{0}=\left(\varepsilon_{\mathrm{eff}}^{t}+\varepsilon_{\mathrm{eff}}^{b}\right), \quad C_{0}=\frac{\varepsilon_{0} L \ln 2}{\pi}, \\
& \varepsilon_{\mathrm{eff}}^{t}=\varepsilon_{\mathrm{eff}}^{b}=\sqrt{\varepsilon_{3}\left[\left(\frac{\varepsilon_{1}+\varepsilon_{2}}{2}\right)+\left(\frac{\varepsilon_{1}-\varepsilon_{2}}{2}\right) \cos 2 \theta\right]},
\end{aligned}
$$

where $2 C_{0}$ is the capacitance of the electrodes in free space, and $\varepsilon_{\text {eff }}^{t}$ and $\varepsilon_{\text {eff }}^{b}$ are the effective dielectric constants for the materials on the top and bottom of the electrodes, respectively. Equation (13) is derived in an assumption that the same anisotropic dielectric is on the top and bottom of the sensor. The exact solution for the capacitance $C_{\theta}$ provides a correction of the coefficient, ln 2 in Eq. (13) or the capacitance $C_{0}$ can be directly measured when it is needed. However, the following analysis does not require the exact knowledge of $C_{0}$, and the estimation provided by Eq. (13) is sufficient.

\section{Dielectrostriction response of an isotropic material under axial deformations}

An elastic dielectric is subjected to three axial strains, $u_{1}, u_{2}$, and $u_{3}$, which are the principal strains in the coordinate system $x_{1} x_{2} x_{3}$ shown in Fig. 5,

$$
\boldsymbol{u}=\left[\begin{array}{ccc}
u_{1} & 0 & 0 \\
0 & u_{2} & 0 \\
0 & 0 & u_{3}
\end{array}\right] .
$$

An initially isotropic dielectric material becomes anisotropic and its dielectric tensor has the same principal coordinate system as the strain tensor $\boldsymbol{u}$ [Eq. (4)]. Principal components of the dielectric tensor, $\varepsilon_{1}, \varepsilon_{2}$, and $\varepsilon_{3}$, are

$$
\begin{aligned}
& \varepsilon_{1}=\varepsilon+\alpha_{1} u_{1}+\alpha_{2}\left(u_{1}+u_{2}+u_{3}\right), \\
& \varepsilon_{2}=\varepsilon+\alpha_{1} u_{2}+\alpha_{2}\left(u_{1}+u_{2}+u_{3}\right),
\end{aligned}
$$

$$
\text { and } \varepsilon_{3}=\varepsilon+\alpha_{1} u_{3}+\alpha_{2}\left(u_{1}+u_{2}+u_{3}\right) \text {. }
$$

Substitution of these principal values into Eq. (13) gives the capacitance response of the planar capacitor sensor to the normal strains,

$$
\begin{aligned}
C_{\theta}= & C_{0}\left\{\varepsilon+\varepsilon_{s}+\frac{1}{2}\left[\left(\frac{1+\cos 2 \theta}{2} u_{1}+\frac{1-\cos 2 \theta}{2} u_{2}\right) \alpha_{1}\right.\right. \\
& \left.\left.+2\left(u_{1}+u_{2}+u_{3}\right) \alpha_{2}\right]\right\}
\end{aligned}
$$

Here $\varepsilon_{s}$ is the dielectric constant of the electrode's substrate. This expression shows that by conducting measurements using different oriented sensors one can extract both electrostriction parameters $\alpha_{1}$ and $\alpha_{2}$. An opposite task can be solved when it is desired: if the electrostriction parameters are known, then dielectrostriction measurements can be used to obtain the magnitudes and directions of the principal strains and/or stresses. In this respect the dielectrostriction effect closely resembles photoelastic effect widely employed in the experimental mechanics for full-field analysis of stresses and strains.

A configuration in Fig. 5 uses two similar planar capacitor sensors whose electrodes are perpendicular to each other. The difference in capacitance of these two sensors is

$$
C_{\theta}-C_{\theta+\pi / 2}=\frac{C_{0}}{2} \alpha_{1}\left(u_{1}-u_{2}\right) \cos 2 \theta .
$$

This expression does not include the parameter $\alpha_{2}$ and excludes the effect of volume deformation. With an additional measurement obtained using the single sensor, the electrostriction parameters $\alpha_{1}$ and $\alpha_{2}$ can be decoupled. Again, an opposite task can be formulated and efficiently solved. Plane rotation of a rosette of perpendicularly oriented sensors varies the capacitance difference as $C_{\theta}-C_{\theta+\pi / 2} \propto \cos 2 \theta$. This enables the principal directions of stresses or strains in the material to be easily determined by finding the orientation of maximum and minimum dielectrostriction response. Such an approach is promising for application in the experimental mechanics and warrants further investigation.

\section{Dielectrostriction study: Uniaxial load}

A dielectric specimen having a dogbone shape is subjected to uniaxial tensile load. Since the tested material is isotropic and linearly elastic, the applied load produces deformations which give rise to an axial elongation strain $u_{1}$ and the lateral contractions $u_{2}=u_{3}\left(=-\nu u_{1}\right.$, where $\nu$ is Poisson's ratio). Two planar capacitor sensors are placed at the specimen surface and are aligned at $\theta=0$ (denoted as $C_{\perp}$ ) and $\theta=\pi / 2$ (denoted as $C_{\|}$) with the load direction (Fig. 6). The principal axis $x_{1}$ is coincident with the load direction, the transverse principal axis $x_{2}$ is in the sensors' plane, and the third principal axis $x_{3}$ is normal to the plane. From Eq. (4), the values of the principal dielectric coefficients $\varepsilon_{1}, \varepsilon_{2}$, and $\varepsilon_{3}$ are

$\varepsilon_{1}=\varepsilon+\left[\alpha_{1}+(1-2 \nu) \alpha_{2}\right] u_{1}$ and 
(a)

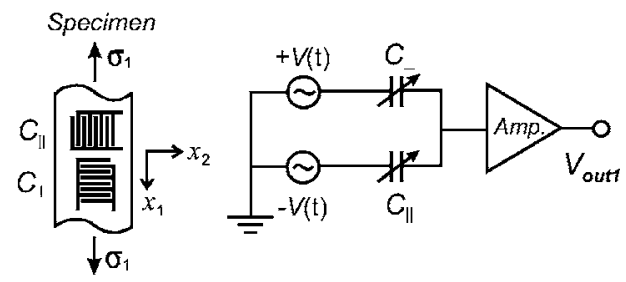

(b) Specimen Dummy

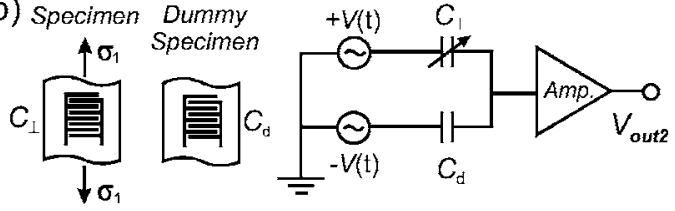

FIG. 6. Two planar capacitors are placed on the specimen surface and are aligned at $\theta=0$ (denoted as $C_{\perp}$ ) and $\theta=\pi / 2$ (denoted as $C_{\|}$) with respect to the load direction. (a) The output $V_{\text {out } 1}$ is proportional to the electrostriction parameter $\alpha_{1}$. (b) Sensor $C_{\perp}$ is combined with a dummy sensor $C_{d}$ to provide the output $V_{\text {out } 2}$ needed to calculate the second electrostriction parameter $\alpha_{2}$.

$$
\varepsilon_{2}=\varepsilon_{3}=\varepsilon+\left[\alpha_{2}-\left(\alpha_{1}+2 \alpha_{2}\right) \nu\right] u_{1} .
$$

The bridge circuit in Fig. 6(a) measures the difference in capacitance, $\Delta C=\left(C_{\perp}-C_{\|}\right)$, which from Eq. (17) can be expressed in terms of the strain $u_{1}$ and the parameter $\alpha_{1}$,

$$
\Delta C\left(=C_{\perp}-C_{\|}\right)=\frac{C_{0}}{2}(1+\nu) \alpha_{1} u_{1}
$$

The output of the capacitor bridge circuit [shown in more detail in Fig. 7(b)] is proportional to the input excitation voltage, $V(t)=V_{i} \sin (\omega t)$, the gain $R$ in the rear amplification stage, and the difference in capacitance, $\Delta C=\left(C_{\perp}-C_{\|}\right)$. Therefore, the output voltage $V_{\text {out }}$ can be expressed as $\left|V_{\text {out }}\right|=\omega R V_{i} \Delta C$ and the output voltage becomes

(a)

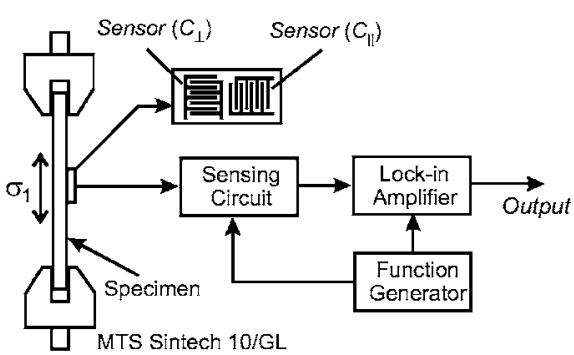

(b)

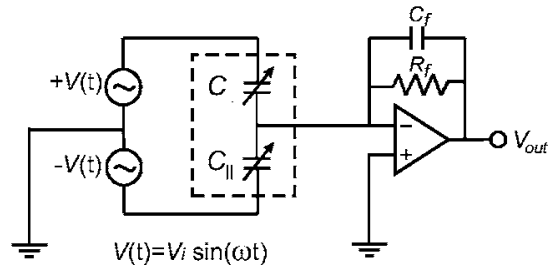

FIG. 7. (a) Two identical sensors having mutually perpendicular interdigitated electrodes are placed in contact with the surface of a tensile-loaded specimen. The dielectrostriction response is measured by a capacitance bridge and conditioned by a lock-in amplifier. (b) The two sensors form the capacitor bridge circuit.

$$
\left|V_{\text {out }}\right|=\left|V_{\text {out } 1}\right|=\omega R V_{i}\left(C_{\perp}-C_{\|}\right)=K(1+\nu) \alpha_{1} u_{1} .
$$

The coefficient $K$ is defined as $K=\left(\omega R V_{i} C_{0}\right) / 2$ in which all parameters can be either calculated or directly measured if needed.

An additional measurement is needed to obtain the other electrostriction parameter, $\alpha_{2}$. The second measuring setup, shown in Fig. 6(b), is similar to that in Fig. 6(a) except that the bridge is now formed by a sensor $C_{\perp}$ and a dummy sensor $C_{d}$. The dummy sensor sees the same environmental conditions as $C_{\perp}$ but not the load. This compensates the output signal for the influence of temperature variations and electromagnetic noise. Similar to Eq. (20), the magnitude of the output signal $\left|V_{\text {out } 2}\right|$ can be obtained by using $C_{d}$ instead of $C_{\|}$as

$$
\left|V_{\text {out } 2}\right|=\omega R V_{i}\left(C_{\perp}-C_{d}\right)=K\left[(1-\nu) \alpha_{1}+2(1-2 \nu) \alpha_{2}\right] u_{1},
$$

where the capacitance $C_{d}$ is given by as $C_{d}$ $=\left[\varepsilon_{0}\left(\varepsilon+\varepsilon_{s}\right) L \ln 2\right] / \pi$. Finally, the electrostriction parameters $\alpha_{1}$ and $\alpha_{2}$ can be expressed through measured outputs,

$$
\begin{aligned}
& \alpha_{1}=\frac{1}{(1+\nu)}\left(\frac{1}{K} \frac{d V_{\text {out1 }}}{d u_{1}}\right), \\
& \alpha_{2}=\frac{1}{2(1-2 \nu)}\left\{\frac{1}{K}\left(\frac{d V_{\text {out } 2}}{d u_{1}}\right)-\alpha_{1}(1-\nu)\right\} .
\end{aligned}
$$

Note that the output voltages $V_{\text {out }}$ and the coefficient $K$ are both similarly proportional to the geometric parameters of the sensor.

\section{EXPERIMENTS}

\section{A. Experimental setup}

A schematic of experimental setup used to make measurements under uniaxial tensile load is shown in Fig. 7. Two sets of perpendicularly oriented interdigitated electrodes are located in close proximity to the surface of the specimen without bonding. A uniaxial tensile load is produced and recorded by the MTS Sintech 10/GL testing machine; the resulting changes in the dielectric properties of the specimen are measured by the capacitor bridge circuit ${ }^{13}$ and conditioned by a SR830 DSP lock-in amplifier. The measurement circuit in Fig. 7(b) uses the input voltage with amplitude, $V_{i}=5 \mathrm{~V}$, and excitation frequency, $f=50 \mathrm{kHz}$. The specimen was loaded at a constant strain rate, $1.27 \mathrm{~mm} / \mathrm{min}(=0.05$ in./min), while its dielectrostriction response was recorded. The polycarbonate specimen has $12.7 \mathrm{~mm}$ width and $3 \mathrm{~mm}$ thickness. The gauge length used to calculate strains is 50 $\mathrm{mm}$. The specimen was injection molded at a mold temperature $T=180^{\circ} \mathrm{F}$, injection speed $v=3 \mathrm{in} / \mathrm{s}$, and pressure $p$ $=2000 \mathrm{psi}$.

\section{B. Results}

\section{Dielectrostriction responses due to uniaxial load}

Figures 8(a) and 9(a) compare the applied stresses and the output voltage of a sensor rosette attached to the polycarbonate specimen for a range of strains. Up to 3\% strain 

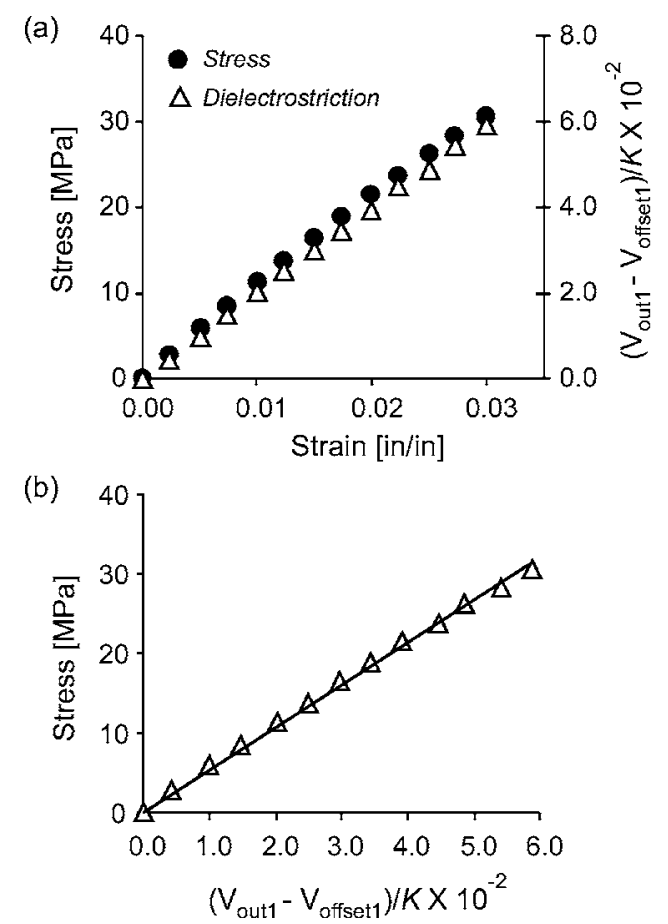

FIG. 8. Two mutually perpendicular sensors, $C_{\perp}$ and $C_{\|}$, form a bridge circuit. The offset voltage $V_{\text {offset }}$ is subtracted from the output signal $V_{\text {outl }}$ and the product is normalized by the coefficient $K$. The linear-elastic limit for the polycarbonate sample is estimated to be $3 \%$ strain. (a) Dielectrostriction/strain response is compared with the stress/strain curve. (b) The same data are presented as the stress-dielectric response.
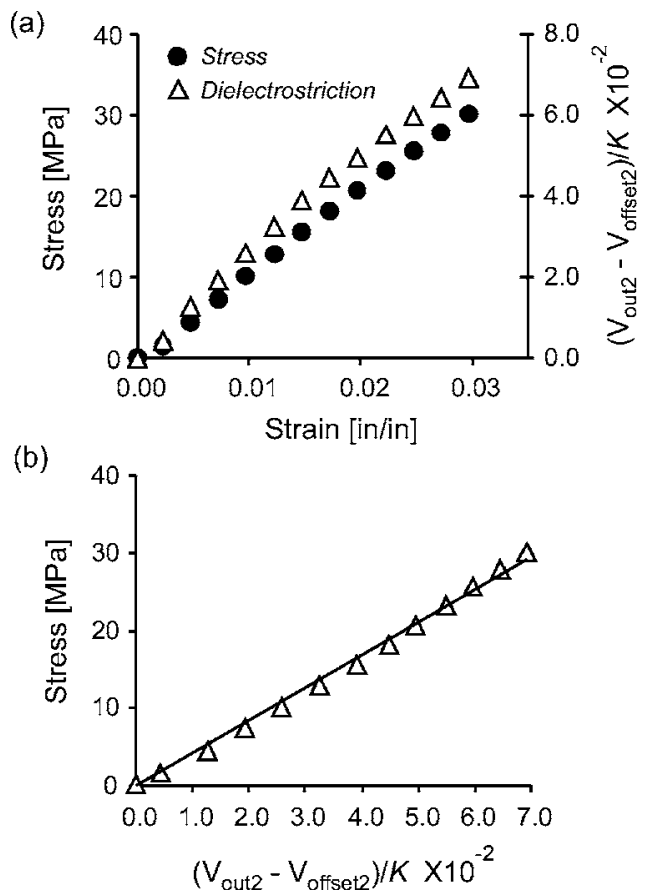

FIG. 9. The specimen from the same batch as that of Fig. 8 is tested using a different sensor rosette. The bridge circuit is formed by two mutually perpendicular sensors $C_{\perp}$ and dummy sensor $C_{d}$. The offset voltage $V_{\text {offset2 }}$ is subtracted from the output signal $V_{\text {out2 }}$ and the product is normalized by the coefficient $K$. (a) Dielectrostriction/strain response is compared with stress/strain curve. (b) The same data are presented as the stress-dielectric response. (a)

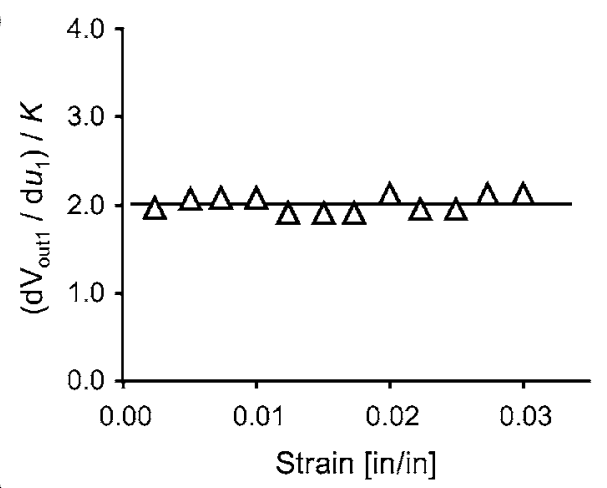

(b)

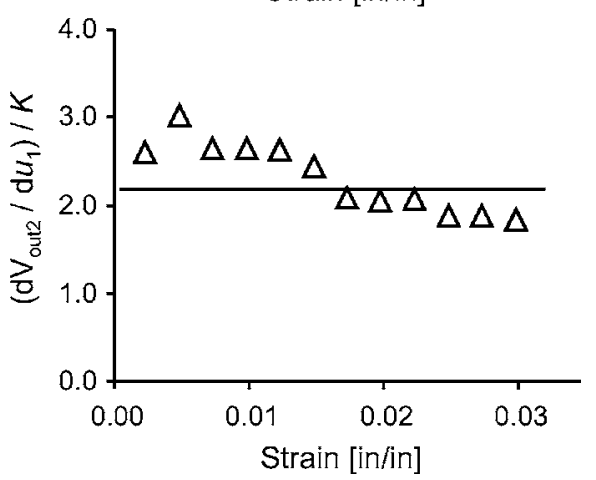

FIG. 10. Derivatives of the data in Figs. 8 and 9 with respect to the strain $u_{1}$ : (a) the average value of $\left(d V_{\text {out } 1} / d u_{1}\right) / K$ is 2.07 , and the (b) average value of $\left(d V_{\text {out } 2} / d u_{1}\right) / K$ is 2.26 .

the specimen is in the linear-elastic region of deformation where stresses are linear with strains. The initially recorded engineering stress $\sigma_{\text {eng }}$ is converted into true stress $\sigma_{\text {true }}$ which accounts for the lateral contraction and is approximated as $\sigma_{\text {true }}=\sigma_{\text {eng }} /\left(1-\nu u_{1}\right)^{2} \approx \sigma_{\text {eng }} /\left(1-2 \nu u_{1}\right)$. According to the analysis in sec. II, the output voltage of a capacitor bridge circuit is proportional to the dielectrostriction response of the specimen. A small mismatch of the capacitances $C_{\perp}$ and $C_{\|}$(or $C_{d}$ ) produces a bridge unbalance and generates the offset voltage $V_{\text {offset }}$. Figures 8 and 9 show the dielectrostriction signal obtained by subtracting the offset voltage from the measured output voltage, and then normalized by the coefficient $K(=10.632 \mathrm{mV})$ which includes the parameters of the measuring circuit and the geometry of the planar capacitor sensor. The measured dielectrostriction response is linear with the strain which is in agreement with the phenomenological relation given by Eq. (4). The raw data from Figs. 8(a) and 9(a) are replotted in the form of stress/dielectrostriction relations in Figs. 8(b) and 9(b). The relationships between the dielectrostriction signals and the stresses are linear. These results illustrate the stress-dielectric rule (SDR) which can lay the foundation for material monitoring using the dielectrostriction effect. ${ }^{11}$

\section{Electrostriction parameters $\alpha_{1}$ and $\alpha_{2}$}

The electrostriction parameters $\alpha_{1}$ and $\alpha_{2}$ determine electrostatic stresses in polarizable materials and are closely related to the material microstructure. ${ }^{8,10-12}$ Equations (22) and (23) indicate that the parameters $\alpha_{1}$ and $\alpha_{2}$ can be obtained by differentiating the normalized outputs ( $V_{\text {out1 }}$ $\left.-V_{\text {offset } 1}\right) / K$ and $\left(V_{\text {out2 }}-V_{\text {offset2 }}\right) / K$, with respect to the defor- 
TABLE I. Comparison of predicted dielectrostriction coefficients for fluids, isotropic solids, and solids having cubic symmetry (all materials have the same dielectric constant). Dielectrostriction response of a cubic material requires the third coefficient $\alpha_{3}$ whose predictions are not currently available.

\begin{tabular}{lcccc}
\hline \hline & & \multicolumn{3}{c}{ Electrostriction parameters } \\
\cline { 3 - 5 } \multicolumn{1}{c}{ Material } & Dielectric constant & $\alpha_{1}$ & $\alpha_{2}$ & $\alpha_{3}$ \\
\hline Newtonian fluid $^{\mathrm{a}}$ & $\varepsilon$ & 0 & $-\frac{1}{3}(\varepsilon-1)(\varepsilon+2)$ & 0 \\
Isotropic solid $^{\mathrm{b}}$ & $\varepsilon$ & $-\frac{2}{5}(\varepsilon-1)^{2}$ & $-\frac{1}{3}(\varepsilon-1)(\varepsilon+2)+\frac{2}{15}(\varepsilon-1)^{2}$ & 0 \\
Cubic solid $^{\mathrm{c}}$ & $\varepsilon$ & $-1.515(\varepsilon-1)^{2}$ & $-\frac{1}{3}(\varepsilon-1)(\varepsilon+2)+0.505(\varepsilon-1)^{2}$ & $\mathrm{NA}^{\mathrm{d}}$ \\
\hline \hline
\end{tabular}

${ }^{\mathrm{a}}$ Reference 1 .

${ }^{\mathrm{b}}$ Reference 12.

${ }^{\mathrm{c}}$ Reference 19.

${ }^{\mathrm{d}} \mathrm{NA}$ stands for not available.

mation $u_{1}$. Figures 10(a) and 10(b) show the derivatives of the experimental data from Figs. 8 and $9 .^{15}$ The value of $\alpha_{1}=1.49$ is obtained by using the average value, $\left(d V_{\text {out } 1} / d u_{1}\right) / K=2.07$, in Fig. 10(a), and Poisson's ratio, $\nu$ $=0.39$. Similarly, the parameter $\alpha_{2}=3.07$ is calculated using the obtained value $\alpha_{1}$ and an estimated average value, $\left(d V_{\text {out } 2} / d u_{1}\right) / K=2.26$, in Fig. 10(b). These measured values of $\alpha_{1}$ and $\alpha_{2}$ are close to the theoretically predicted ones for isotropic dielectrics ${ }^{12}$ [see Eq. (5)]. For the dielectric constant of polycarbonate, $\varepsilon=2.9-3.0$, the calculated values of electrostriction parameters are $\left|\alpha_{1}\right|=1.44-1.6$ and $\left|\alpha_{2}\right|$ $=2.62-2.80$. Some disagreement with the theory could arise because the proposed theory assumes isotropy but injection molding flow can introduce some anisotropy in the tested specimens.

\section{DISCUSSION}

The results herein show that the dielectrostriction response is linear with stress under elastic deformation, which resembles the well-known photoelastic effect. Following the stress-optical rule (SOR) for photoelastic behavior, we defined this relationship as the SDR. ${ }^{11}$ This resemblance becomes obvious if one recognizes that the magnetic permeability of polymers, $\mu$, is close to one $(\mu \approx 1)$ and replaces $\varepsilon$ with $\sqrt{ } n$ (note $n^{2}=\varepsilon \mu$ ). Therefore, birefringence can be considered as a manifestation of dielectrostriction in the optical range of electromagnetic spectrum. However, while only electronic polarization is involved in the birefringence, all polarization mechanisms [e.g., interfacial (MaxwellWagner), dipole, ionic, and electronic polarization mechanisms] contribute to the dielectrostriction. Consequently, while optical measurements are limited to transparent materials, dielectrostriction is applicable to both transparent and opaque materials. Moreover, the measurements can be conducted over wide range of frequencies of the electric field, which makes dielectrostriction more suitable for in-line processing and material health monitoring.

Dielectrostriction measurements are useful for materials study. According to Shkel and Klingenberg, ${ }^{10,12}$ the dielectrostriction response of a suspension is determined by the pair-distribution function of the suspended particles. Even materials showing the same dielectric properties, determined by a single dielectric constant $\varepsilon$, will provide vastly different results for $\alpha_{1}$ and $\alpha_{2}$ (see Table I). In a static situation, both isotropic fluid and solid materials might have the same dielectric constants. However, a fluid would remain isotropic during any deformations and the coefficient $\alpha_{1}$, therefore, is always zero. The only dielectrostriction effect observed in fluids and gases is due to the variations of the dielectric constant with the density. Materials having cubic structure are traditionally considered as a lattice approximation for isotropic materials. However, the description of the photoelastic effect in such materials requires three coefficients (see Ref. 2, page 252. Due to analogy between photoelasticity and dielectrostriction, the dielectrostrictive response of cubic materials also requires the third coefficient $\alpha_{3}$ microscopic models for which are not yet available. (Following the notation used here, the electrostriction coefficient in cubic materials can be introduced as $\varepsilon_{i j}=\varepsilon \delta_{i j}+\alpha_{1} u_{i j}+\alpha_{2} u_{k k} \delta_{i j}$ $+\alpha_{3} u_{l m} d_{l} d_{m} d_{i} d_{j}$, where $d_{i}$ is the unit vector in the lattice direction.) Dielectrostrictive behavior of anisotropic materials is even more complicated than that of isotropic. The general phenomenological approach to dielectrostriction in anisotropic solids is outlined by Shkel and Klingenberg. ${ }^{10}$

This study introduces a solid-state capacitance sensing method which is able to monitor the dielectrostriction phenomenon. ${ }^{11,14,16}$ This technique uses change in dielectric properties of a material instead of traditional capacitance measurements relying on a geometric variation of the electrodes. The solid-state capacitor sensor incorporating a dielectrostriction sensing mechanism provides a more robust structure than the conventional vacuum-gap capacitance sensor and is easy to be manufactured with the selection of various dielectric materials potentially optimized by dynamic properties and environmental requirements. Tan and Castner ${ }^{17}$ and Arshak et al. ${ }^{18}$ employed parallel-plate capacitors to detect the change in dielectric properties of the uniaxially loaded material. However, such parallel-plate configuration has inherent difficulties with the boundary conditions, as described in Sec. II. The planar sensor approach to dielectrostriction measurement introduced in this study can minimize the effect of geometric variations and directly obtain the dielectric response due to deformations. In addition, by rotating two sets of mutually perpendicular planar capacitor sensors, one is able to determine the principal directions of stress/strain without knowing their absolute values. Potential applications of dielectrostriction measurements include in-line material health monitoring, nondestructive evaluation 
(NDE), self-sensing and actuating smart materials, and microstructure studies of dielectrics. However, the full potential of dielectrostriction phenomenon for applications is not completely explored at this stage.

\section{CONCLUSION}

The traditional method for studying electrostriction through field-induced deformation is subject to concern because the exact boundary conditions at the material-electrode interfaces are unknown. Different testing conditions can result in different values of electrostrictive coefficients. The present study is believed to adopt a more appropriate approach where electrostriction is described through the fieldinduced stresses. Electrostriction stress is one of the components in field-induced stresses. It depends on the effect of strain on the polarization of dielectrics which is described by electrostriction parameters. General thermodynamic considerations of dielectric solids identify such parameters as strain derivatives of the dielectric coefficient. Strain/dielectric response of materials is called dielectrostriction. Studying dielectrostriction phenomena presents specific challenges, e.g., controlling mechanical boundary conditions for thin-film specimens which are traditionally used for dielectric measurements. A planar capacitor sensor is proposed here for studying dielectrostriction and measurement of dielectric responses due to deformations. A significant feature of the planar capacitor sensing mechanism proposed here is that it needs no mechanical contact with the specimen. Based on this sensing method, we show that the two sets of perpendicularly oriented interdigitated capacitor sensors are useful for studying the dielectrostriction effect, electrostriction parameters, and material health monitoring. Employing this configuration, one is able to obtain the dielectrostriction responses caused by uniaxial deformation and extract the magnitudes of the electrostriction parameters $\alpha_{1}$ and $\alpha_{2}$. These measured values are in good agreement with the theory for isotropic solid dielectrics, [Eq. (5)]. Dielectrostriction studies can also provide useful information about material structures and enable applications to material health monitoring. However, at this stage, the dielectrostriction phenomenon which describes the stress/strain-dielectric relationship in various material structures remains not fully understood and warrants further investigation. Our future efforts will include improving these sensing techniques and devices.

\section{ACKNOWLEDGMENTS}

The authors would like to thank Professor R. Rowlands, UW-Madison, and Professor D. Matthys, Marquette University, Milwaukee, for helpful discussions. This work was supported in part by AFOSR Grant No. FA9550-04-1-0238 and NSF Grant No. CMS-0437890.

${ }^{1}$ J. A. Stratton, Electromagnetic Theory (McGraw-Hill, New York, 1941).

${ }^{2}$ J. F. Nye, Physical Properties of Crystals: Their Representation by Tensors and Matrices (Oxford University Press, New York, 1985).

${ }^{3}$ A. F. Devonshire, Adv. Phys. 3, 85 (1954).

${ }^{4}$ V. Sundar and R. E. Newnham, Ferroelectrics 135, 431 (1992).

${ }^{5}$ Z. Y. Meng and L. E. Cross, J. Appl. Phys. 57, 488 (1985).

${ }^{6}$ Y. M. Shkel and D. J. Klingenberg, J. Appl. Phys. 80, 4566 (1996).

${ }^{7}$ F. M. Guillot and J. Jarzynski, J. Acoust. Soc. Am. 110, 2980 (2001).

${ }^{8}$ L. D. Landau, E. M. Lifshitz, and L. P. Pitaevskii, Electrodynamics of Continuous Media, 2nd ed. (Butterworth-Heinenann, Oxford, 1984) Vol. 8.

${ }^{9}$ C. Rinaldi and H. Brenner, Phys. Rev. E 65, 036615 (2002).

${ }^{10}$ Y. M. Shkel and D. J. Klingenberg, J. Rheol. 43, 1307 (1999).

${ }^{11}$ Y. Peng, Y. M. Shkel, and G. H. Kim, J. Rheol. 49, 297 (2005).

${ }^{12}$ Y. M. Shkel and D. J. Klingenberg, J. Appl. Phys. 83, 7834 (1998).

${ }^{13}$ L. K. Baxter, Capacitive Sensors: Design and Applications (IEEE, New York, 1997).

${ }^{14}$ G. H. Kim and Y. M. Shkel, J. Intell. Mater. Syst. Struct. 13, 479 (2002).

${ }^{15}$ S. C. Chapra, Numerical Methods for Engineers, 2nd ed. (McGraw-Hill, New York, 1988).

${ }^{16}$ Y. M. Shkel and N. J. Ferrier, IEEE/ASME Trans. Mechatron. 8, 318 (2003).

${ }^{17}$ H. S. Tan and T. G. Castner, Phys. Rev. B 23, 3983 (1981).

${ }^{18}$ K. I. Arshak, D. McDonagh, and M. A. Durcan, Sens. Actuators, A 79, 102 (2000).

${ }^{19}$ R. A. Anderson, Phys. Rev. B 33, 1302 (1986). 